\title{
Ultrasmall Porous Silica Nanoparticles with Enhanced Pharmacokinetics for Cancer Theranostics
}

Carolina A. Ferreira ${ }^{1} \neq$, Shreya Goel ${ }^{2} \neq$, Emily B. Ehlerding', Zachary T. Rosenkrans ${ }^{4}$, Dawei

Jiang 5 , Tuanwei Sun ${ }^{5}$, Eduardo Aluicio-Sarduy ${ }^{3}$, Jonathan W. Engle ${ }^{3}$, Dalong Ni ${ }^{5}{ }^{*}$, Weibo $\operatorname{Cai}^{1,2,3,4,5 *}$

Department of ${ }^{1}$ Biomedical Engineering, ${ }^{2}$ Materials Science \& Engineering, ${ }^{3}$ Medical Physics, ${ }^{4}$ Pharmaceutical Sciences, and ${ }^{5}$ Radiology, University of Wisconsin - Madison, Madison, Wisconsin 53705, United States. 

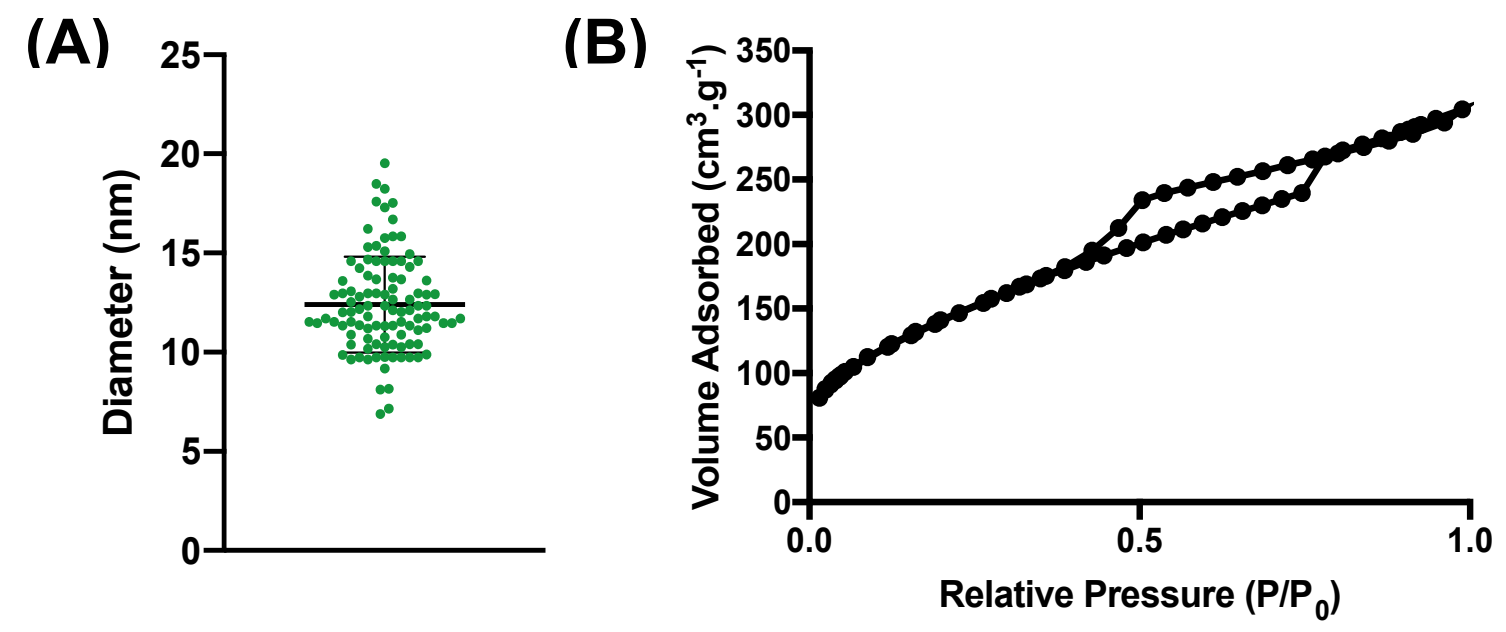

(C)

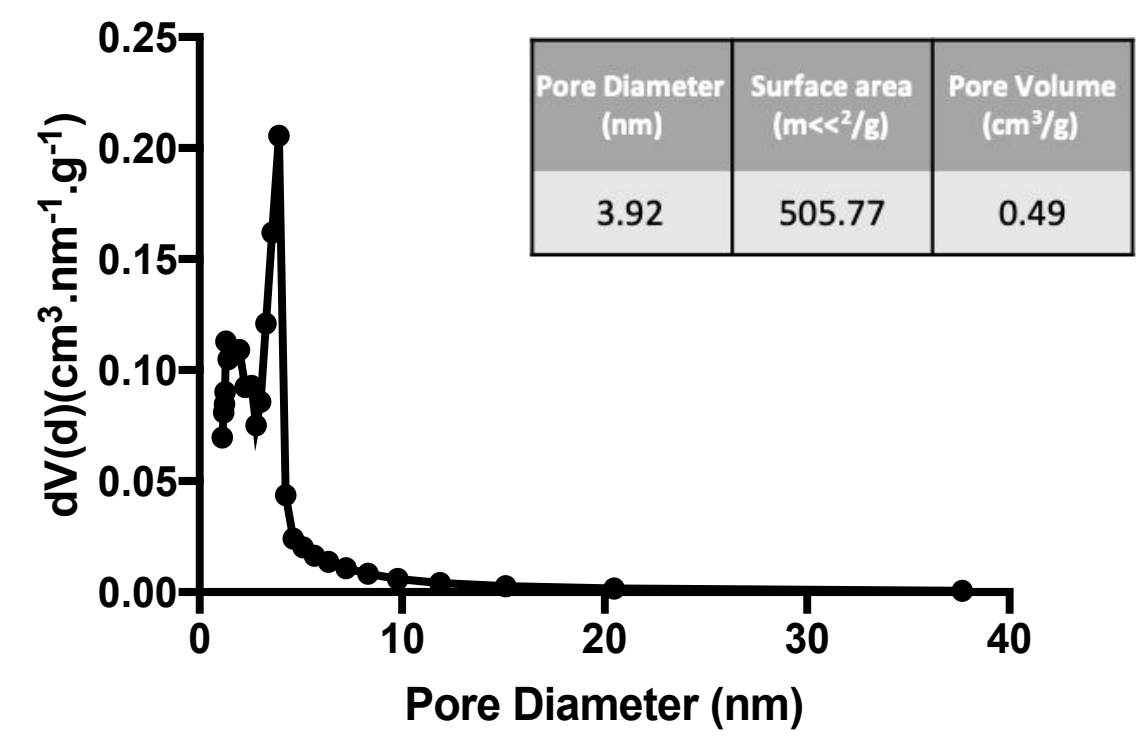

Figure S1. Nanoparticle characterization. (A) Average particle diameter of UPSN, determined from TEM images. At least 100 particles of 10 fields of view were analyzed. Black lines represent mean \pm SD. (B) Nitrogen adsorption isotherms revealing a hysteresis loop characteristic of porous materials. (C) Pore Diameter density distribution. Insert with numerical information of averaged pore diameter, surface area and pore volume calculated from BET analysis. 
Table S1. Groups and Doses for the Therapeutic Study

\section{Treatment Group Therapeutic Agent}

${ }^{90}$ Y-DOTA-UPSN $\quad \sim 5.5 \mathrm{MBq}{ }^{90}$ Y-DOTA-UPSN

${ }^{90} \mathrm{Y}$-DOTA $\quad \sim 5.5 \mathrm{MBq}{ }^{90} \mathrm{Y}$-DOTA

UPSN only $\quad 50 \mu \mathrm{L}$ of as-prepared UPSN

PBS only $\quad 50 \mu \mathrm{L}$ of PBS

Table S2. Experiments and choice of isotope

\begin{tabular}{cc}
\hline Experiment & Radiolabeled agent \\
\hline PET Imaging & ${ }^{86}$ Y-DOTA-UPSN \\
\hline Dosimetric Studies & ${ }^{86}$ Y-DOTA-UPSN \\
\hline Cerenkov Luminescence Imaging & ${ }^{90}$ Y-DOTA-UPSN \\
\hline Therapy & ${ }^{90}$ Y-DOTA-UPSN \\
\hline Toxicity Studes & \\
\hline 90 & \\
\hline
\end{tabular}

Table S3. Radiolabeling Stability Studies. Tabulated average values (percentage) obtained from iTLC.

\begin{tabular}{ccccc}
\hline Media & $\mathbf{1 5} \mathbf{~ m i n}$ & $\mathbf{4 h}$ & $\mathbf{2 4 h}$ & $\mathbf{4 8 h}$ \\
\hline PBS & 93.2 & 92.7 & 89.9 & 85.1 \\
\hline Serum & 92.9 & 91.3 & 88.1 & 78.3 \\
\hline
\end{tabular}


(A)

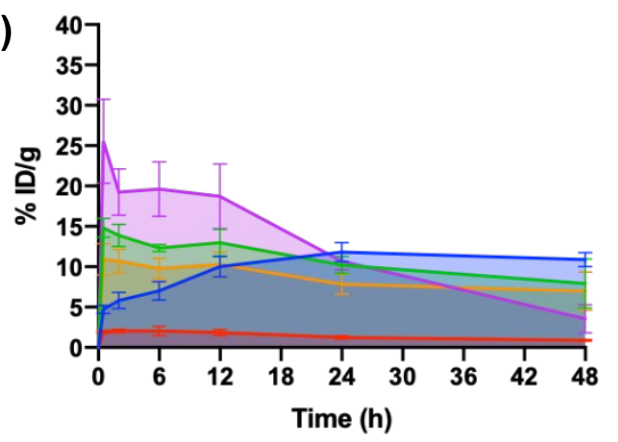

- Tumor

- Muscle

- Liver

- Blood Pool

- Spleen
(B)

\begin{tabular}{|c|c|}
\hline Tissue & AUC $\left(\% \mid \mathrm{D} \cdot \mathrm{g}^{-1} \cdot \mathbf{h}\right)$ \\
\hline Tumor & 489.2 \\
\hline Muscle & 67.62 \\
\hline Liver & 510.1 \\
\hline Blood Pool & 579.9 \\
\hline Spleen & 406.6 \\
\hline
\end{tabular}

Figure S2. Area-under-curve (A) graph and (B) values in animals injected with ${ }^{86}$ Y-DOTA-UPSN monitored over 48 hours. Values indicate exposure (dose per gram of tissue) obtained from experimental observations.

(A)

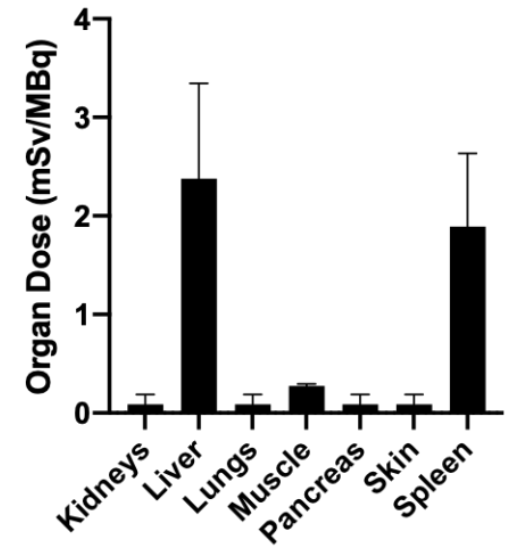

(B)

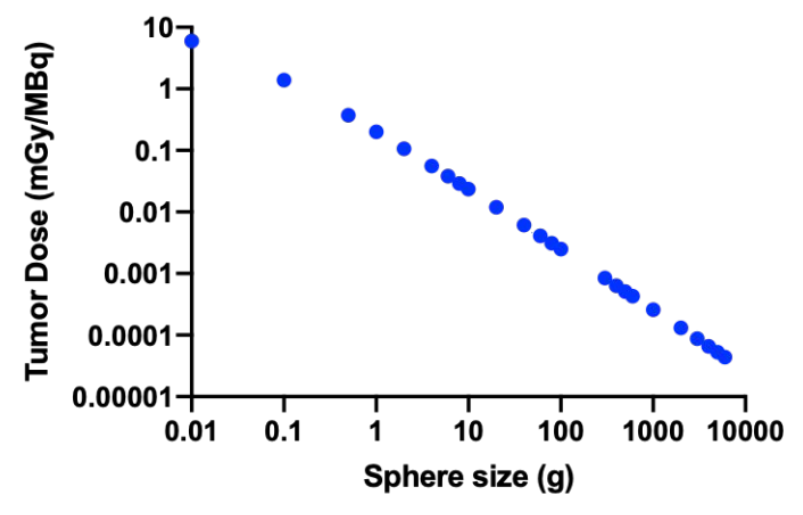

Figure S3. Dosimetry of ${ }^{90}$ Y-DOTA-UPSN, extrapolated from ${ }^{86}$ Y-DOTA-UPSN PET Data. (A) Doses to major organs. (B) Tumor doses calculated through dose-to-sphere modeling. 
(A)

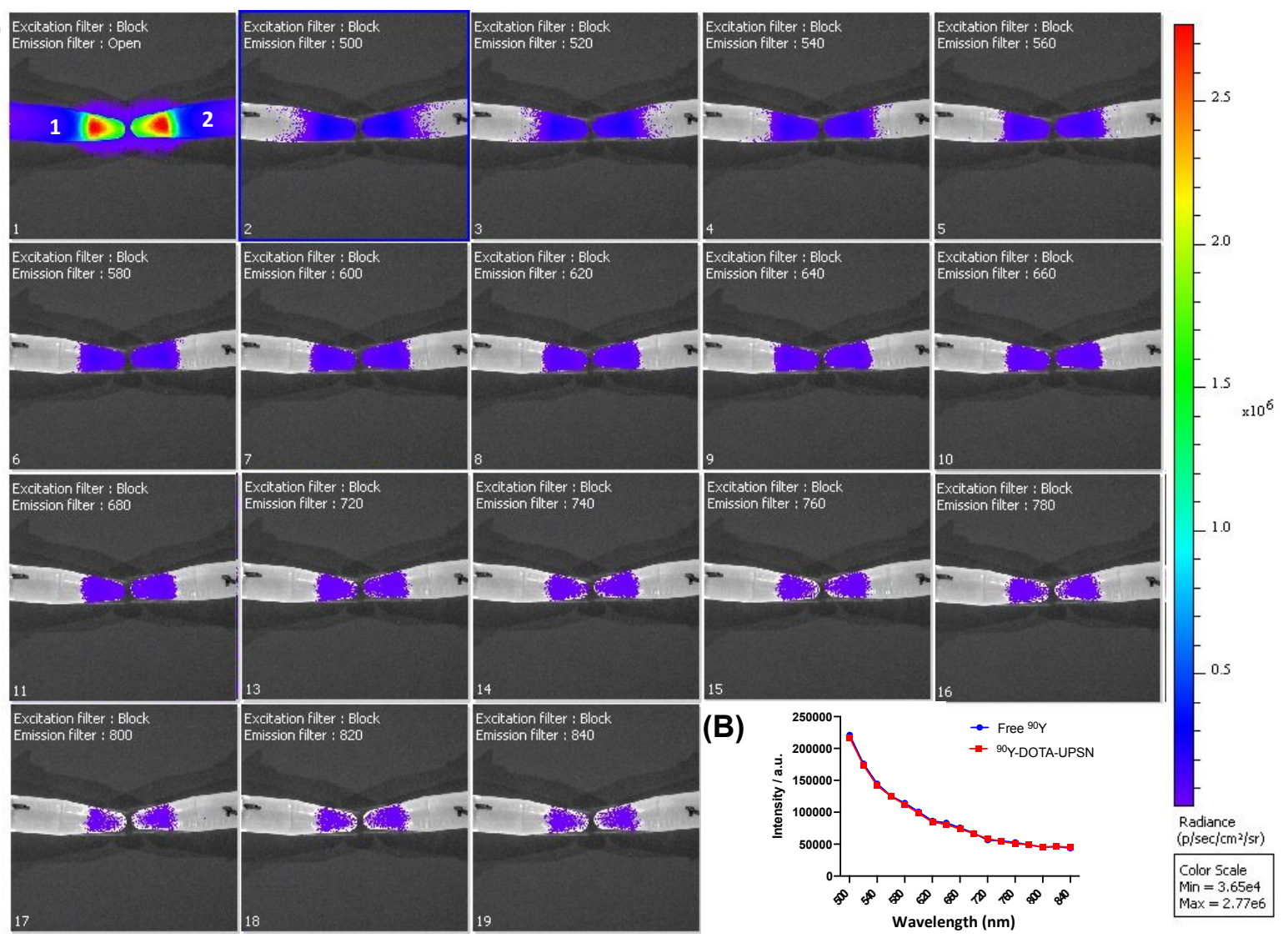

Figure S4. (A) Cerenkov luminescence emissions of samples at different wavelengths. 1: free ${ }^{90} \mathrm{Y}$ $(15 \mu \mathrm{Ci}) .2:{ }^{90} \mathrm{Y}$-DOTA-UPSN $(15 \mu \mathrm{Ci})$. (B) Spectral emissions of free ${ }^{90} \mathrm{Y}$ and ${ }^{90} \mathrm{Y}-\mathrm{DOTA}-\mathrm{UPSN}$ on the IVIS Imaging system. 
(A)

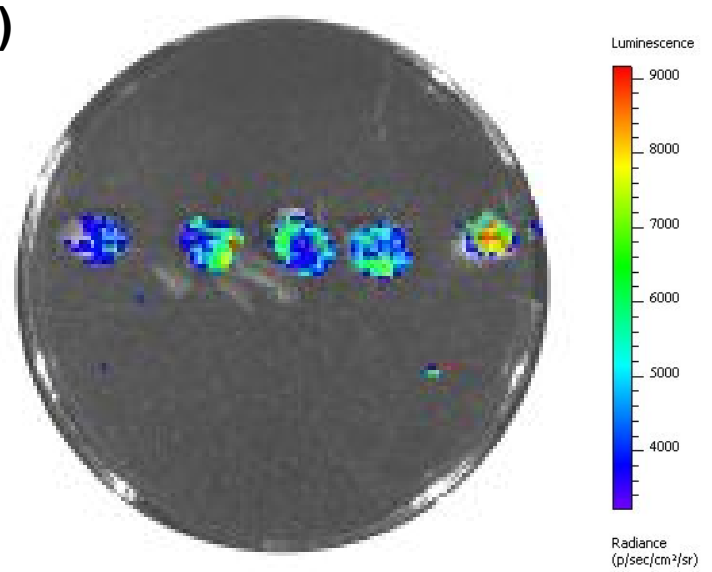

(B)

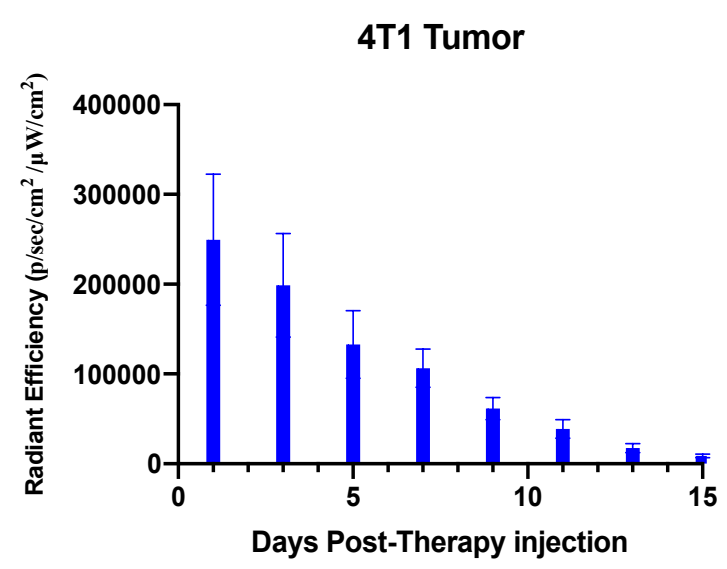

Figure S5. (A) Ex vivo CLI of tumor tissue collected from 5 mice that received ${ }^{90}$ Y-DOTA-UPSN.

(B) In vivo tumor uptake values from CLI images as Radiant Efficiency.

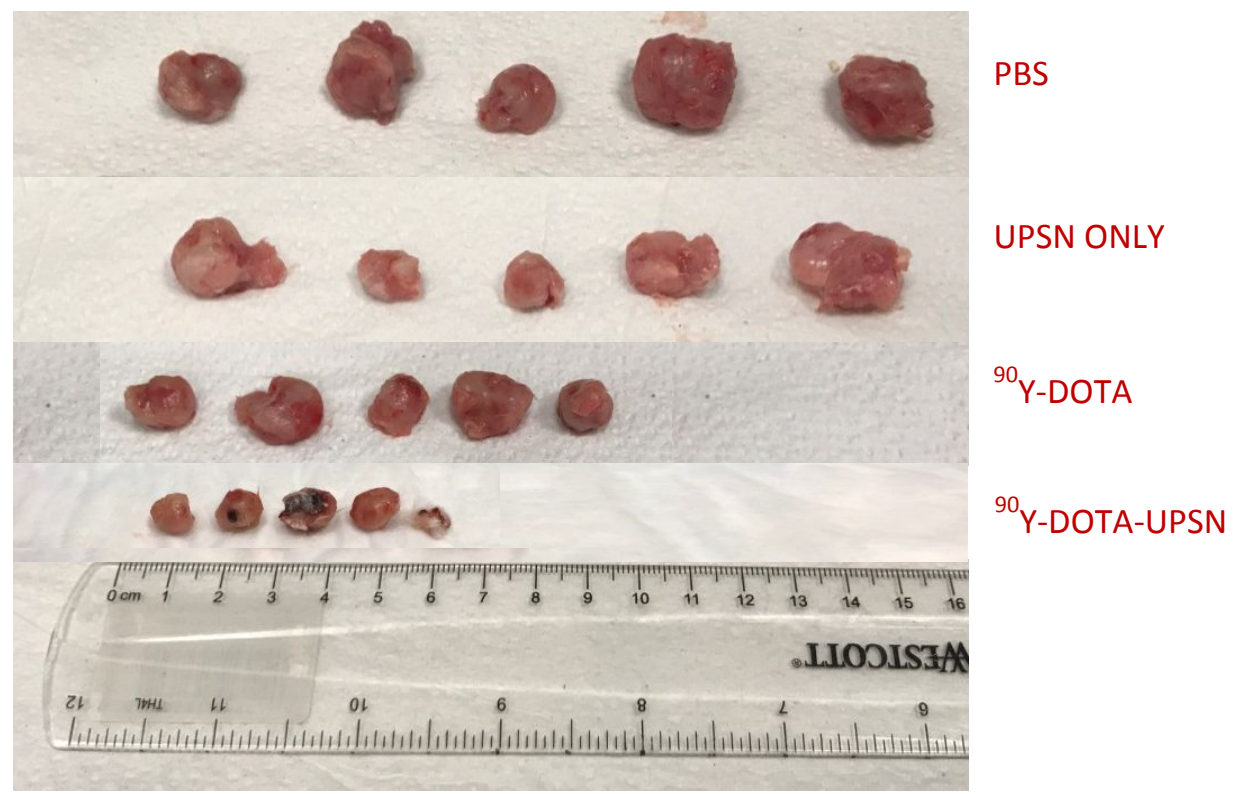

Figure S6. Images of tumors excised at the end of the study (Day 15 p.i) or after reaching an endpoint, demonstrating a clear difference in size. 


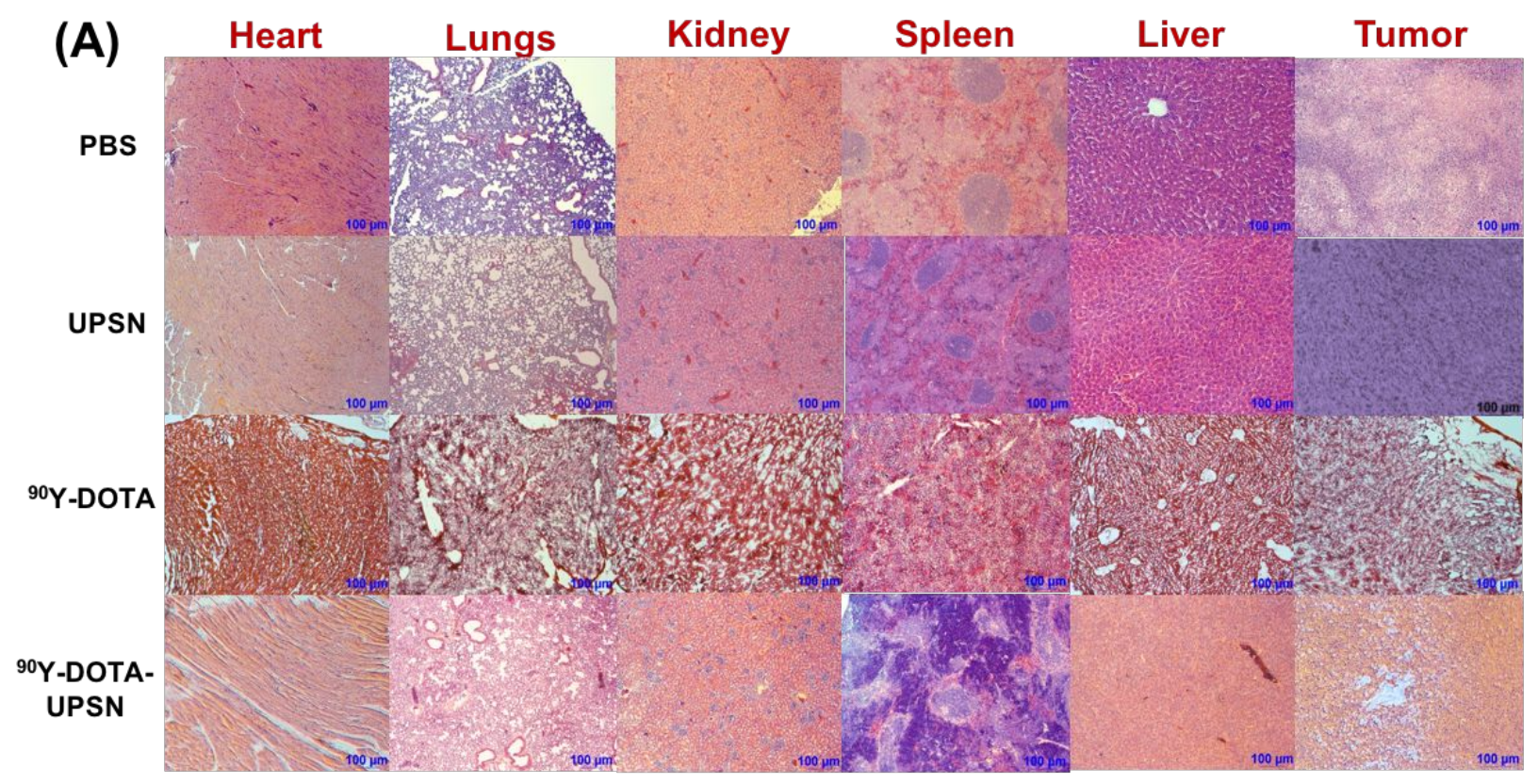

(B)

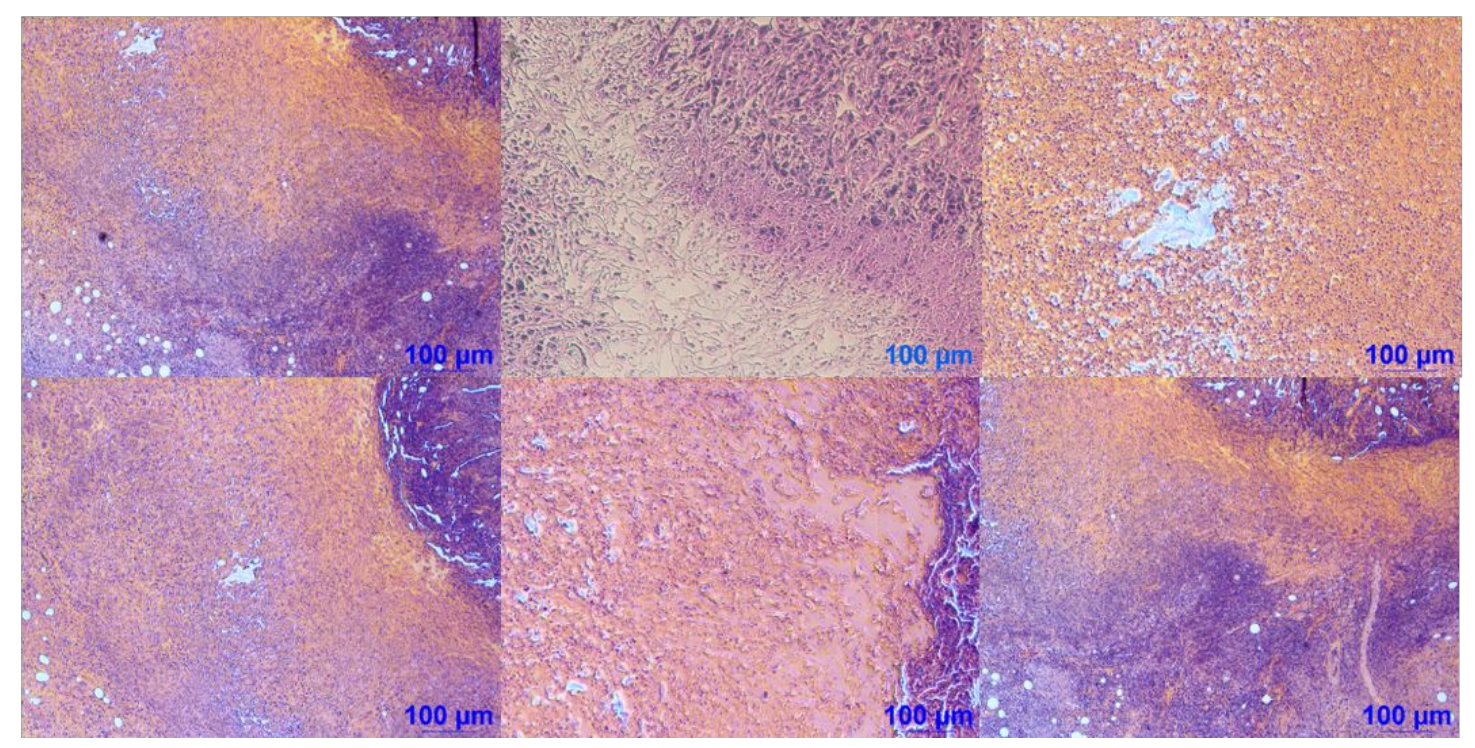

Figure S7. Histological evaluation through Haemotoxylin and Eosin (H\&E) staining of tissues.

(A) Representative images of H\&E staining of different tissues from all therapeutic groups at 15 days p.i. Only tissues from mice that received 90Y-DOTA presented morphologic alterations as signs of potential organ toxicity. (B) H\&E staining of tumor tissues excised from animals 15 days post-injection of ${ }^{90} \mathrm{Y}$-DOTA-UPSNs with several areas of necrosis revealing therapeutic agent anti-tumor effects 


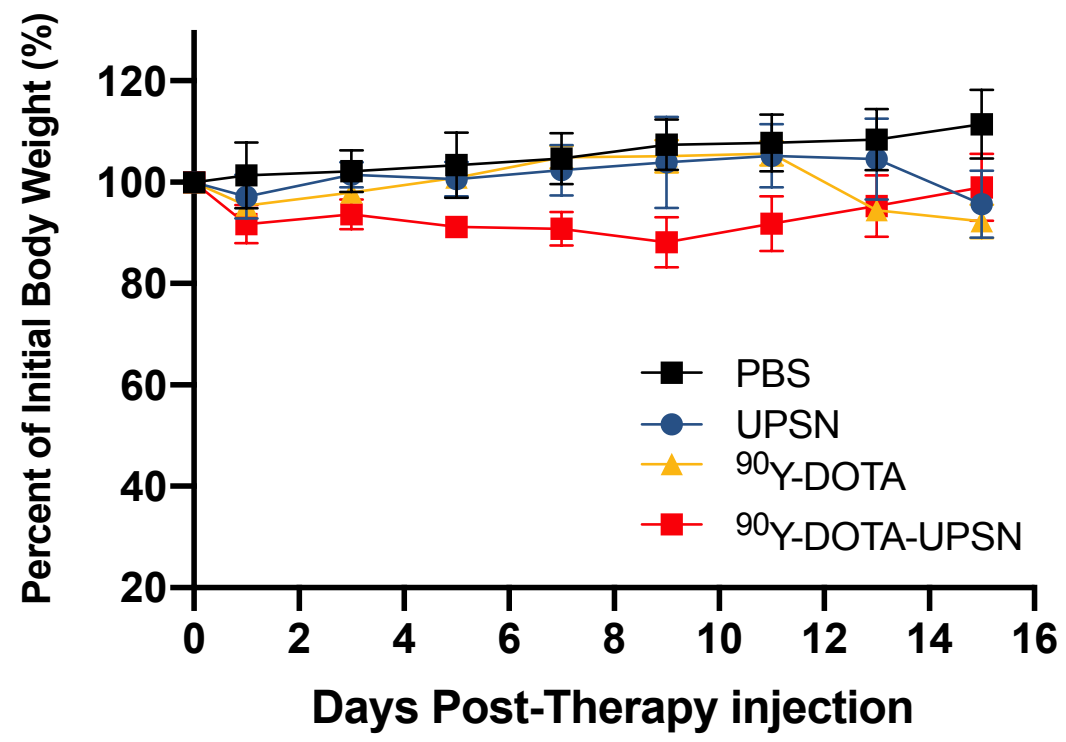

Figure S8. Body weights measured every other day, as relative values from initial. None of the mice reached a body weight below humane endpoint $(<80 \%)$, revealing low overall toxicity to administered agents.

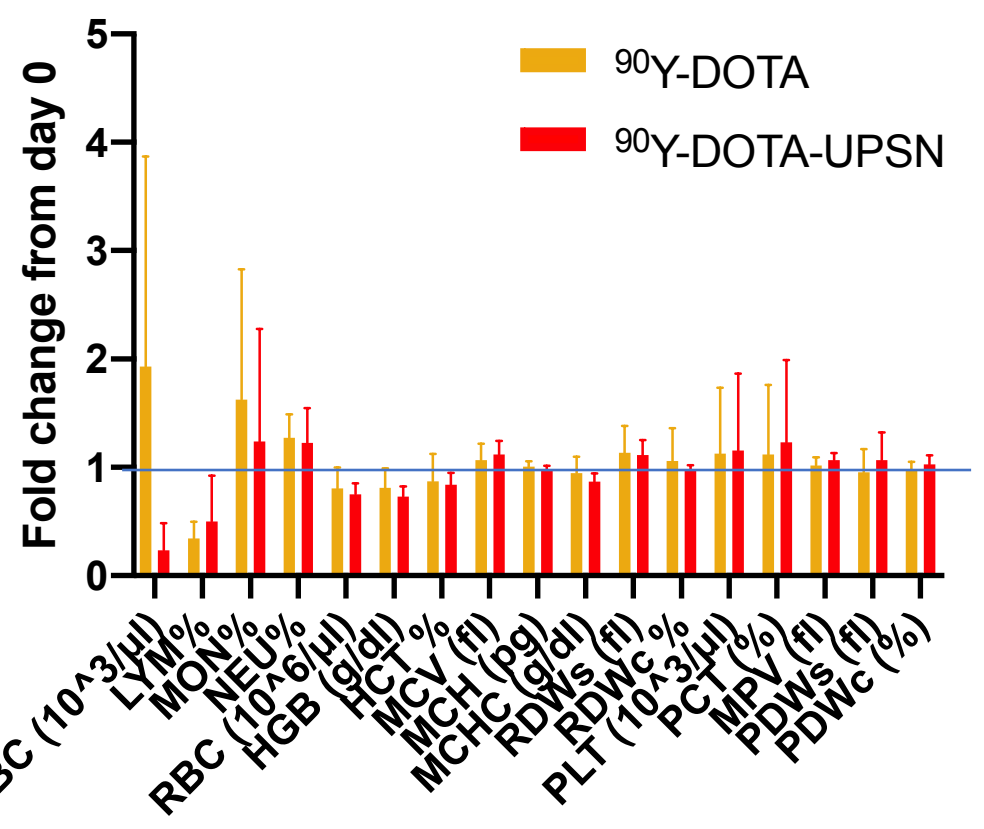

Figure S9. Complete blood count analysis from mice injected with ${ }^{90} \mathrm{Y}$-DOTA or ${ }^{90} \mathrm{Y}$-DOTAUPSN at the end of the study (day 15), showed as relative values from day 0. 


\section{Experimental Section}

\section{Cell Culture and Animal Models}

All animal-based experiments were conducted under an approved protocol by the University of Wisconsin-Madison Institutional Animal Care and Use Committee. 4T1 murine mammary cancer cells (ATCC) were cultured using recommended conditions (RPMI 1640 medium with 10\% FBS, $\left.37{ }^{\circ} \mathrm{C}, 5 \% \mathrm{CO} 2\right)$ until approximately $75 \%$ confluence. All animal-based experiments were conducted under an approved protocol by the University of Wisconsin-Madison Institutional Animal Care and Use Committee. Approximately 1x106 4T1 cells were implanted in the lower flank of 4-5 weeks old female mice (BALB/c purchased from Envigo). Tumor-bearing animals were included in the studies approximately 7 days after xenograft implantation.

\section{Synthesis and Characterization of UPSN}

UPSN synthesis was carried out through a slight variation of a "one-pot" method first demonstrated by Ma et.al. ${ }^{1}$. Briefly, $83.82 \mathrm{mg}(0.23 \mathrm{mmol})$ of hexadecyltrimethylammonium bromide (CTAB), $2 \mathrm{~mL}$ of $\mathrm{H}_{2} \mathrm{O}$ and $20 \mu \mathrm{L}$ of $2 \mathrm{M}$ ammonia solution were added to a $10 \mathrm{~mL}$ flask, followed by addition of $8 \mathrm{~mL} \mathrm{H}_{2} \mathrm{O}$. The sample was stirred at $30^{\circ} \mathrm{C}$ for $0.5 \mathrm{~h}$ followed by addition of $65.45 \mathrm{mg}$ of Tetramethyl orthosilica (TMOS) (0.43 mmol). 24 hours later, $105 \mathrm{mg}(0.21 \mathrm{mmol})$ of PEG-silane (MW: $500 \mathrm{Da}$; 5-9 ethylene glycol units) was added to the sample and stirred for another 24 hours at $30^{\circ} \mathrm{C}$. Then, $1.66 \mathrm{mg} \quad(0.0043 \quad \mathrm{mmol})$ of N-3[(amino(polypropylenoxy)]aminopropyltrimethoxysilane $\left(\mathrm{NH}_{2}-\mathrm{PPO}-\right.$ silane; 350-400 Da, 3-4 propylenoxy units) was added to the mixture and stirred for another $2 \mathrm{~h}$ at $30^{\circ} \mathrm{C}$ to obtain nanoparticles with $\mathrm{NH}_{2}$ functional groups on the surface. The molar ratio of the reaction was 1 TMOS: 0.53 CTAB: 0.093 ammonium hydroxide: 0.49 PEG-silane: $0.01 \mathrm{NH}_{2}$-PPO-silane: 1292 
$\mathrm{H}_{2} \mathrm{O}$. The temperature was then increased to $80^{\circ} \mathrm{C}$ for an additional $24 \mathrm{~h}$. The sample was then transferred to a dialysis membrane with a $10 \mathrm{kDa}$ cut off and dialyzed in acid solution $(1: 1: 0.007$ water, ethanol and acetic acid) for $24 \mathrm{~h}$ to remove CTAB. The process was repeated three times, followed by dialysis in water for another three times for $24 \mathrm{~h}$ each.

Transmission Electron Microscopy (TEM) samples were imaged on a FEI T12 microscope, operated at an accelerating voltage of $120 \mathrm{kV}$. Nitrogen adsorption-desorption isotherms were measured at $77 \mathrm{~K}$ using a Quantachrome Nova 4000e system after the removal of organic template. The brunauer-Emmett-Teller (BET) method was applied for surface area calculation and pore size distribution by the Barrett, Joyner and Halenda (BJH) method. Particle size distribution using Dynamic Light Scattering and zeta potential analysis were performed on Nano-Zetasizer (Malvern Instruments Ltd.).

\section{${ }^{89 / 90}$ Y Labeling of UPSN}

DOTA was chosen as a chelator for ${ }^{86 / 90} \mathrm{Y}$. The nanoparticles were conjugated with DOTA in a similar manner as described previously ${ }^{2}$. Briefly, $1 \mathrm{~mL}$ of UPSN-PEG-NH $\mathrm{N}_{2}$ was reacted with approximately $45 \mathrm{nmol}$ of $\mathrm{p}-\mathrm{SCN}-\mathrm{Bn}$-DOTA at basic $\mathrm{pH}$ to obtain UPSN-PEG-NH $\mathrm{N}_{2}$-DOTA (UPSN-DOTA). The UPSN-DOTA conjugate was purified with PD-10 size exclusion columns to remove unconjugated DOTA molecules. $74 \mathrm{MBq}$ of ${ }^{86} \mathrm{YCl} 3$ in $300 \mu \mathrm{L}$ of NaOAc buffer (pH 6.5) was added to UPSN-DOTA. The mixture was incubated at $37^{\circ} \mathrm{C}$ for 2 hours. The sample was then purified through a PD-10 column (GE Healthcare) system and eluted with PBS. Radiolabeling yields were measured via Instant thin layer chromatography (iTLC), using silica impregnated paper as the stationary phase and 50mM EDTA as the mobile phase, at different time points and 
after purification. iTLC chromatograms were developed using a cyclone phosphor-plate imager reader and analyzed with Optiquant software.

\section{PET Imaging, Biodistribution Studies and Dosimetry}

4T1 tumor-bearing mice $(\mathrm{n}=5)$ were intravenously injected with 2-3 MBq of ${ }^{86}$ Y-DOTA-UPSN and submitted to serial PET scans in Inveon PET/CT scanner (Siemens). PET scans of 100 million coincidence events per mouse were obtained at $0.5,2,6,12,24$, and $48 \mathrm{~h}$ post-injection (p.i.). Regions-of-interest (ROI) were calculated from the images using the Inveon Research Workspace (Siemens). At the end of the imaging study, major organs were and had their radioactivity measured through a gamma counter (PerkinElmer). Quantitative results are presented as percentage injected dose per gram of tissue $(\% \mathrm{ID} / \mathrm{g})$.

Using OLINDA/EXM software, ROI data was used to extrapolate organ doses of ${ }^{90} \mathrm{Y}$-DOTAUPSN to an adult female, as well as tumor doses through dose-to-sphere modeling.

\section{Therapeutic Administration}

Same methodology described for ${ }^{86} \mathrm{Y}$-labeling of tracers was carried out to obtain ${ }^{90} \mathrm{Y}$-labeled UPSNs. Treatment groups injected into of $4 \mathrm{~T} 1$ tumor-bearing ( $\mathrm{n}=5$ per group) can be found at table S1. Selected dosage was based on previously reported studies ${ }^{3,4}$.

\section{Treatment Monitoring and Cerenkov Imaging}


Mice included in the therapeutic study were monitored every other day for body weight measurements collection. Tumor dimensions were also measured using a digital caliper and its volume is calculated from the length $\times$ width $^{2}$ formula. Data represented as relative tumor volume increase derived from the tumor volume at that timepoint divided by tumor volume at the first day of therapy injection. Concurrently, ${ }^{90} \mathrm{Y}$-therapeutics were monitored in vivo using an IVIS optical imaging system (PerkinElmer) for up to 15 days which is more than five half-lives of ${ }^{90} \mathrm{Y}\left(\mathrm{t}_{1 / 2}=\right.$ $64 \mathrm{~h}$ ). For that, anesthetized animals were placed in lateral decubitus position and 60 second open filter exposures were acquired., Humane end points were set as a tumor volume that increase three times its original size, body weight value below $80 \%$ of initial, or if general health was compromised.

\section{Toxicity Evaluation and Tissue Analysis}

Blood samples were collected before administration of therapeutics and at 15 days p.i. for blood count analysis using an Abaxis VetScan HM5 Hematology analyzer $(n=5)$. At the end of the study, relevant liver and kidney function parameters (ALT, AST, CRE, and BUN) were also submitted for evaluation at the University of Wisconsin - Madison School of Veterinary Medicine. At the end of the study major organs were collected, submitted to hematoxylin and eosin (H\&E) staining and analyzed using a Nikon Eclipse Ti microscope.

\section{Statistical Analysis}

Data is presented as mean \pm standard deviation. For comparisons between groups, a two-sided Student's $\mathrm{t}$ test was performed in which a $\mathrm{p}<0.05$ was considered statistically significant. 


\section{References}

1. Ma, K.; Sai, H.; Wiesner, U. Ultrasmall sub-10 nm near-infrared fluorescent mesoporous silica nanoparticles. J Am Chem Soc 2012, 134, (32), 13180-3.

2. Goel, S.; Chen, F.; Hong, H.; Valdovinos, H. F.; Hernandez, R.; Shi, S.; Barnhart, T. E.; Cai, W. VEGF(1)(2)(1)-conjugated mesoporous silica nanoparticle: a tumor targeted drug delivery system. ACS Appl Mater Interfaces 2014, 6, (23), 21677-85.

3. Ehlerding, E. B.; Ferreira, C. A.; Aluicio-Sarduy, E.; Jiang, D.; Lee, H. J.; Theuer, C. P.; Engle, J. W.; Cai, W. (86/90)Y-Based Theranostics Targeting Angiogenesis in a Murine Breast Cancer Model. Mol Pharm 2018, 15, (7), 2606-2613.

4. Ehlerding, E. B.; Lacognata, S.; Jiang, D.; Ferreira, C. A.; Goel, S.; Hernandez, R.; Jeffery, J. J.; Theuer, C. P.; Cai, W. Targeting angiogenesis for radioimmunotherapy with a (177)Lulabeled antibody. Eur J Nucl Med Mol Imaging 2018, 45, (1), 123-131. 